List your meeting here:

Following is a list of microscopy related meetings and courses. The editors would greatly appreciate input to this list via the electronic submission form under "events/ other microscopy events" at the MSA webpage: www.microscopy.org. We will gladly add hypertext links to the notice on the web and insert a listing of the meeting in the next issue. Please furnish the following information (any additional information provided will be edited as required and printed on a space available basis):

- Meeting/Course name

- Meeting/Course dates

- Meeting/Course topic or short description

- Sponsoring society/organization/university

- Contact person

- Telephone number

- Fax number

- Email address

- URL for website

\section{Meetings 2019}

\section{Netherlands Electron Microscopy \\ Infrastructure Meeting}

November 6, 2019

Geertekerk, The Netherlands

Contact: Mima Malcicka: miriama. malcicka@ffund.nl

\section{EMBL in the USA 2019}

\section{Symposium: The Future of}

Transcontinental Science

November 8-9, 2019

Stanford, CA

www.embl.it/aboutus/alumni/eventsnetworks/local-chapters/usa/96_ usa_2019/index.html

\section{ISTAFA: 2019}

November 10-14, 2019

Portland, OR

www.asminternational.org/web/istfa-2019/ home

\section{NZCM 2019 - 29 th $N e w$ Zealand}

\section{Conference on Microscopy}

November 11-14, 2019

Hamilton, New Zealand

www.ivvy.com.au/event/Y7FLHK/home. html
Innovation in Material

Science \& Technology

November 17-19, 2019

Singapore

https://symposium.acs.org/2019/singapore

\section{International Thin Films}

Conference (TACT2019)

November 17-20, 2019

Taipei, Taiwan

http://tact2019.conf.tw/site/page.

aspx?pid=901\&sid=1245\&lang=en

\section{One-Day flowcytometryUK \\ Meeting 2019}

November 21, 2019

Cambridge, UK

www.rms.org.uk/discover-engage/eventcalendar/one-day-flowcytometryukmeeting-2019.html

\section{Advanced Microscopy \\ Techniques for Plant-microbe \\ Interaction Analysis \\ November 25-29, 2019 \\ Tulln, Austria \\ www.plant-microbe-microscopy.com/ program}

\section{MRS 2019 Fall Meeting \\ December 1-6, 2019 \\ Boston, MA \\ www.mrs.org/fall2019}

\section{BPACRCM 2019 - Biological and Pharmaceutical Applications of CRS Microscopy \\ December 2-4, 2019 \\ Odense, Denmark \\ www.conferencemanager.dk/CRS- microscopy 2019}

\section{ASCB 2019 Annual Meeting \\ December 7-11, 2019 \\ Washington, CA \\ www.ascb.org/meetings-events/future-ascb- meetings}

\section{$8^{\text {th }}$ International Conference on Mechanics of Biomaterials and Tissues}

December 15-19, 2019

Waikoloa Beach, Hawaii

www.elsevier.com/events/conferences/ international-conference-on-mechanicsof-biomaterials-and-tissues

\section{Courses 2019}

\section{Scanning Electron Microscopy: Advanced Imaging Practices \\ November 4-5, 2019 \\ Westmont, IL \\ Sponsor: Hooke College of Applied Sciences www.mccrone.com/courses/sem-advanced- imaging}

Biological and Pharmaceutical Applications of CRS Microscopy

December 2-4, 2019

Odense, Denmark

www.conferencemanager.dk/CRSmicroscopy 2019 


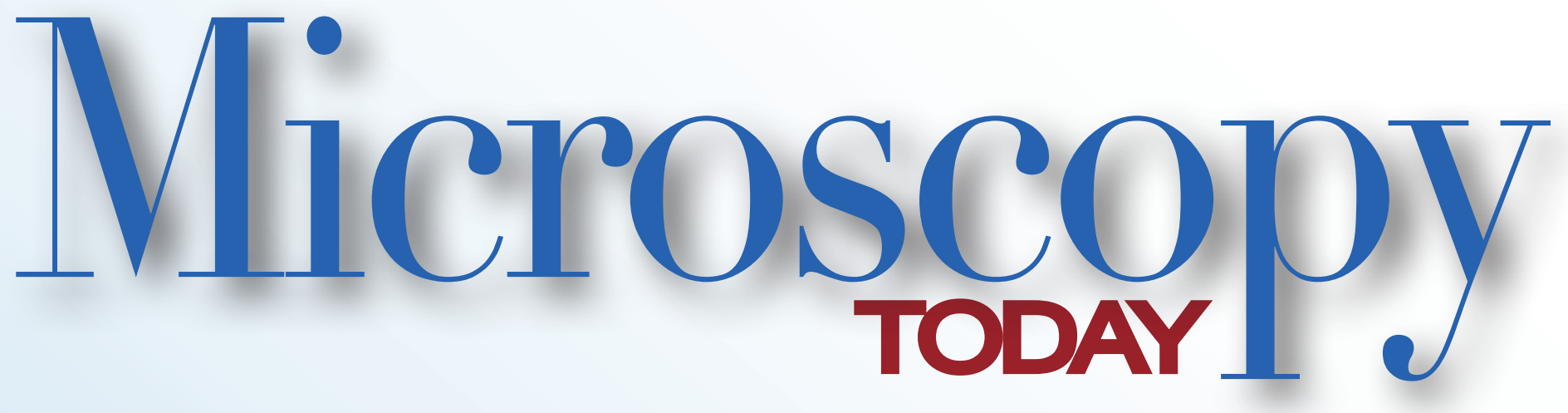

Innovation Awards
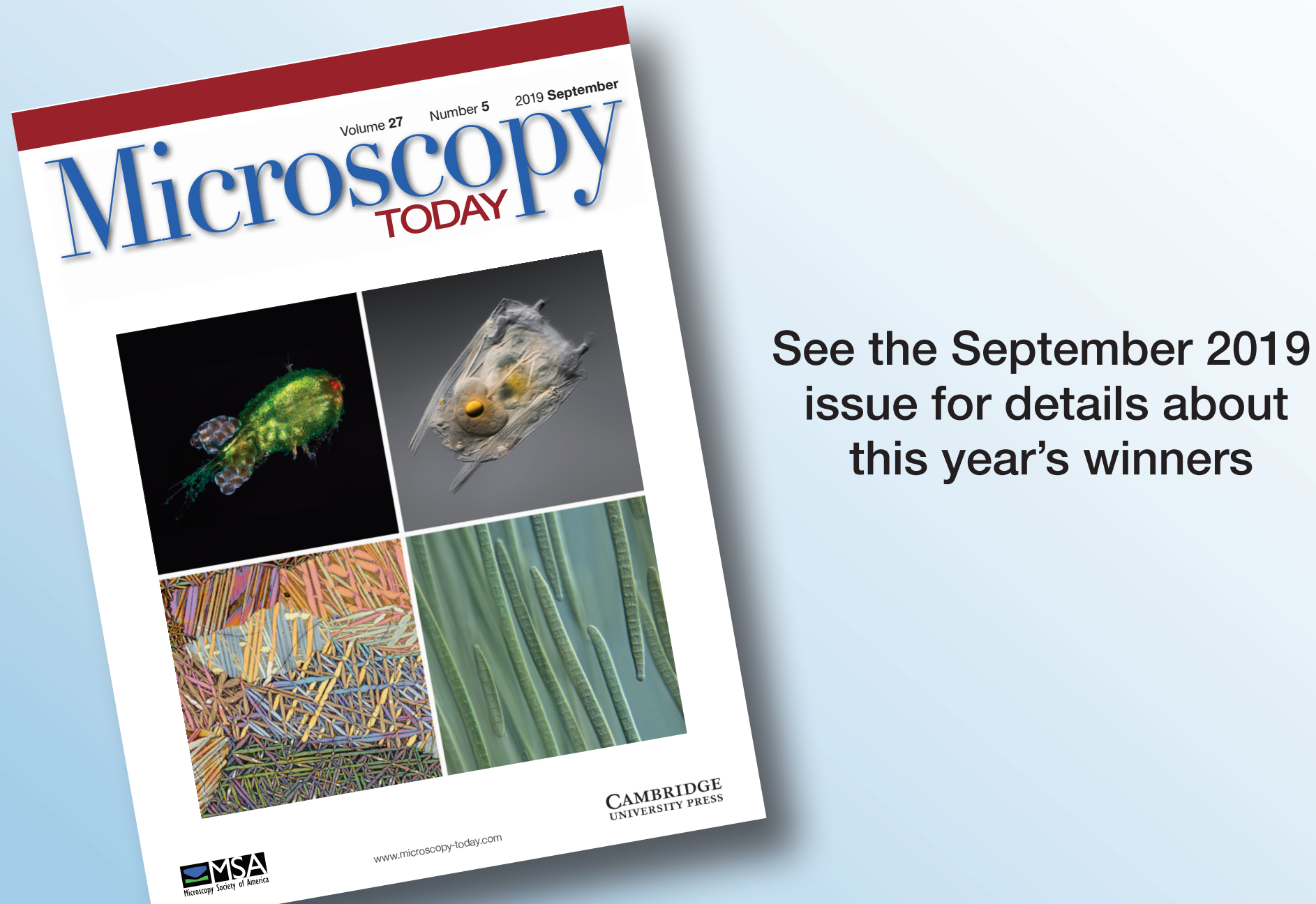

Request application forms by email: charles.lyman@lehigh.edu Next deadline - March 21, 2020 
Meetings 2020

UK Light Microscopy Facility Meeting 2020

January, 6-7, 2020

Birmingham, UK

www.rms.org.uk/discover-engage/event-calendar/ uk-light-microscopy-facility-meeting-2020.html

EM-UK 2020

January 9-10, 2020

Plymouth, UK

www.rms.org.uk/discover-engage/eventcalendar/em-uk-2020.html

\section{Liquid Phase Electron Microscopy Gordon Research Conference \\ January 26-31, 2020 \\ Lucca (Barga), Italy \\ www.grc.org/_resources/common/userfiles/file/ GRC\%20at\%20renaissance\%20tuscany.pdf}

\section{APMC-2020 - 12th Asia-Pacific \\ Microscopy Conference \\ February 3-7, 2020 \\ Hyderabad, India \\ www.apmc12.in}

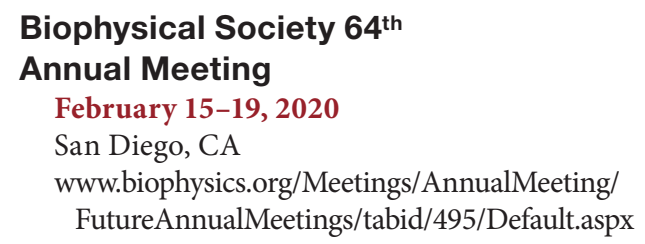

ACMM $26-26^{\text {th }}$ Australian Conference on Microscopy and Microanalysis

February 15-20, 2020

Canberra, Australia

www.acmm26.org/welcome

Future Materials 2020

- Materials Science \&

Nanotechnology Conference

February 26-28, 2020

Lisbon, Portugal

Contact: rishi.k@materialseurope.com

http://materialsconference.yuktan.com

\section{United States and Canadian}

Academy of Pathology (USCAP)

Annual Meeting 2020

February 29-March 06, 2020

Los Angeles, CA

www.emedevents.com/c/ medical-conferences-2020/

united-states-and-canadian-academy-ofpathology-uscap-annual-meeting-2020

AIMS 2020 - AIMS Annual Conference

March 20, 2020

Tempe, AZ

Sponsor: Arizona Imaging and Microanalysis Society

http://azmicroscopy.org/events
ACS Annual Conference.

Macromolecular Chemistry:

The Second Century

March 22-26, 2020

Philadelphia, PA

www.acs.org/content/acs/en/meetings/

national-meeting/abstract-submission.html

FOM2020 - Focus on Microscopy 2020

April 5-8, 2020

Osaka, Japan

http://focusonmicroscopy.org

2020 MRS Spring Meeting \& Exhibit

April 13-17, 2020

Phoenix, AZ

www.mrs.org/spring2020

ICMCTF'20- International

Conference on Metallurgical

Coatings \& Thin Films

April 26-May 1, 2020

San Diego, CA

www2.avs.org/conferences/ICMCTF/2019/

index.htm

$3^{\text {th }}$ Anniversary World

Congress on Biosensors

May 26-29, 2020

Busan, Korea

www.elsevier.com/events/conferences/worldcongress-on-biosensors

\section{ULTRAPATH XX}

June 7-12, 2020

Amsterdam, The Netherlands

https://ultrapath.org

\section{Gordon Research Seminar - Three \\ Dimensional Electron Microscopy \\ June 20-21, 2020 \\ Castelldefels, Spain \\ www.grc.org/find-a-conference}

\section{Gordon Research Conference - Three}

Dimensional Electron Microscopy

June 21-26, 2020

Castelldefels, Spain

www.grc.org/find-a-conference

$8^{\text {th }}$ International Workshop

on Focused Electron Beam-

Induced Processing (FEBIP)

July 8-10, 2020

Glasgow, UK

http://febip.org

FENS Forum 2020

July 11-15, 2020

Glasgow, Scotland

Email: forum2020@fens.org

www.fens.org/News-Activities/Calendar/ Meetings/2020/07/FENS-Forum-2020
Microscopy \& Microanalysis 2020

August 2-6, 2020

Milwaukee, WI

Email: nicoleguy@conferencemanagers.com www.microscopy.org

DXC 2020: Denver X-Ray

Conference 2020

August 3-7, 2020

Rockville, MD

www.dxcicdd.com

emc2020: 17 $7^{\text {th }}$ European

Microscopy Congress

August 23-28, 2020

Copenhagen, Denmark

www.emc2020.eu

$16^{\text {th }}$ International Congress

of Histochemistry and

Cytochemistry (ICHC)

August 30-September 2, 2020

Prague, Czech Republic

http://ichc2020.com

ASM Annual Meeting (IMAT): Solving

Global Materials Challenges

September 14-17, 2020

Cleveland, $\mathrm{OH}$

www.asminternational.org/web/imat

\section{$4^{\text {th }}$ Annual NSH Symposium/}

\section{Convention}

October 16-21, 2020

Reno, NV

Sponsor: National Society for

Histotechnology

www.histoconvention.org/futuredates.cfm

\section{Neuroscience 2020}

October 24-28, 2020

Washington, DC

Sponsor: Society for Neuroscience

www.sfn.org

AVS $67^{\text {th }}$ International

Symposium \& Exhibition

October 25-30, 2020

Denver, CO

www.avs.org/symposium

2020 MRS Fall Meeting \& Exhibit

November 29-December 3, 2020

Boston, MA

www.mrs.org/fall2020

\section{ASCB 2020 Annual Meeting}

December 5-9, 2020

Philadelphia, PA

www.ascb.org/meetings-events/future-ascbmeetings 


\section{Courses 2020}

\section{Biological TEM: A Complete Picture}

January 14-16, 2020

Hatfield, PA

Sponsor: Electron Microscopy Sciences

www.emsmicroscopyacademy.com/productpage/biotem-jan20

\section{Resin Electron Microscopy}

January 15-17, 2020

Utrecht, The Netherlands

www.cellbiology-utrecht.nl/electronmicroscopy-courses/resin-electronmicroscopy.html

\section{Cryosectioning and Immuno- \\ Electron Microscopy}

January 20-23, 2020

Utrecht, The Netherlands

www.cellbiology-utrecht.nl/electronmicroscopy-courses/cryosectioning-andimmuno-electron-microscopy.html

\section{X-Ray Microanalysis}

January 21-23, 2020

Hatfield, PA

Sponsor: Electron Microscopy Sciences www.emsmicroscopyacademy.com/productpage/xray-jan 20

\section{Automated and Rapid Specimen \\ Processing for Electron Microscopy}

February 18-20, 2020

Hatfield, PA

Sponsor: Electron Microscopy Sciences www.emsmicroscopyacademy.com/productpage/auto-feb20

\section{Materials Ultramicrotomy}

February 24, 2020 (Beginner)

February 25-27, 2020 (Some Experience)

Hatfield, PA

Sponsor: Electron Microscopy Sciences www.emsmicroscopyacademy.com/productpage/materials-feb20

\section{Introduction to Microscopy \\ Techniques}

March 3-5, 2020

Hatfield, PA

Sponsor: Electron Microscopy Sciences www.emsmicroscopyacademy.com/productpage/intro-mar20

\section{Biological SEM}

March 17-19, 2020

Hatfield, PA

Sponsor: Electron Microscopy Sciences www.emsmicroscopyacademy.com/productpage/biosem-mar20

\section{Cryo SEM}

March 24-26, 2020

Hatfield, PA

Sponsor: Electron Microscopy Sciences www.emsmicroscopyacademy.com/productpage/cryosem-mar20

Aurion Immunogold Silver Staining April 15-17, 2020

Hatfield, PA

Sponsor: Electron Microscopy Sciences www.emsmicroscopyacademy.com/productpage/immunogold-apr20

\section{Cryosectioning/Immunogold}

April 20-24, 2020

Hatfield, PA

Sponsor: Electron Microscopy Sciences www.emsmicroscopyacademy.com/productpage/cryoimmuno-apr20

\section{Introduction to Microscopy \\ Techniques}

May 19-21, 2020

Hatfield, PA

Sponsor: Electron Microscopy Sciences www.emsmicroscopyacademy.com/productpage/intro-may 20

\section{X-Ray Microanalysis}

May 26-28, 2020

Hatfield, PA

Sponsor: Electron Microscopy Sciences www.emsmicroscopyacademy.com/productpage/xray-may20

\section{Lehigh Microscopy School: \\ SEM and TEM}

May 31-June 5, 2020

Bethlehem, PA

www.lehigh.edu/microscopy

\section{Microscopy: The Complete}

Image - June

June 15-26, 2020

Hatfield, PA

Sponsor: Electron Microscopy Sciences www.emsmicroscopyacademy.com/productpage/completeimage-jun 20

\section{Microscopy: The Complete \\ Image - July \\ July 13-24, 2020}

Hatfield, PA

Sponsor: Electron Microscopy Sciences www.emsmicroscopyacademy.com/productpage/completeimage-jul20

\section{Microscopy: The Complete Image - August}

August 10-21, 2020

Hatfield, PA

Sponsor: Electron Microscopy Sciences www.emsmicroscopyacademy.com/productpage/completeimage-aug20

\section{Introduction to Microscopy Techniques}

September 15-17, 2020

Hatfield, PA

Sponsor: Electron Microscopy Sciences www.emsmicroscopyacademy.com/productpage/intro-sep20

\section{Materials Ultramicrotomy}

September 21, 2020 (Beginner)

September 22-24, 2020 (Some Experience) Hatfield, PA

Sponsor: Electron Microscopy Sciences www.emsmicroscopyacademy.com/productpage/materials-sep20

\section{Cryo SEM}

October 13-15, 2020

Hatfield, PA

Sponsor: Electron Microscopy Sciences www.emsmicroscopyacademy.com/productpage/cryosem-oct 20

\section{Biological SEM}

October 20-22, 2020

Hatfield, PA

Sponsor: Electron Microscopy Sciences www.emsmicroscopyacademy.com/productpage/biosem-oct 20

\section{Aurion Immunogold Silver Staining}

November 10-12, 2020

Hatfield, PA

Sponsor: Electron Microscopy Sciences www.emsmicroscopyacademy.com/productpage/immunogold-nov20

\section{Biological TEM}

November 17-19, 2020

Hatfield, PA

Sponsor: Electron Microscopy Sciences www.emsmicroscopyacademy.com/productpage/biotem-nov20

\section{Meetings 2021}

\section{MRS Spring Meeting \& Exhibit}

April 19-23, 2021

Seattle, WA

www.mrs.org/spring2021

EMAS 2021 - 17 $7^{\text {th }}$ European Workshop on Modern Developments and Applications in Microbeam Analysis

May 16-20, 2021

Krakow, Poland

www.microbeamanalysis.eu/events/ event/60-emas-2021-17th-europeanworkshop-on-modern-developments-andapplications-in-microbeam-analysis 
mmc2021: Microscience

Microscopy Congress 2021

July 5-8, 2021

Manchester, UK

www.mmc-series.org.uk

\section{Microscopy \& Microanalysis 2021}

August 1-5, 2021

Pittsburgh, PA

Email: nicoleguy@conferencemanagers.com

www.microscopy.org

\section{Microscopy Conference}

(Dreiländertagung) MC 2021

August 22-26, 2021

Vienna, Austria

https://asem.at/events

\section{Microscopy Conference 2021}

August 23-27, 2021

Vienna, Austria

www.microscopy-conference.de

\section{$4^{\text {th }}$ Annual NSH Symposium/ \\ Convention}

September 16-23, 2021

Omaha, NE

Sponsor: National Society for

Histotechnology

www.histoconvention.org/futuredates.cfm

\section{Neuroscience 2021}

November 13-17, 2021

Chicago, IL

Sponsor: Society for Neuroscience

www.sfn.org

\section{MRS Fall Meeting}

November 28-December 3, 2021

Boston, MA

www.mrs.org/fall2021

\section{ASCB 2021 Annual Meeting}

December 11-15, 2021

San Diego, CA

www.ascb.org/meetings-events/future-ascbmeetings

\section{Meetings 2022}

\section{FENS Forum 2022}

July 9-13, 2022

Paris, France

Email: forum2022@fens.org

www.fens.org/News-Activities/Calendar/

Meetings/2022/07/FENS-Forum-2022
Microscopy \& Microanalysis 2022

July 31-August 4, 2022

Portland, OR

Email: nicoleguy@conferencemanagers.com

www.microscopy.org

\section{IMC20: 20 th International Microscopy Congress}

September 25-30, 2022

Busan, Korea

https://uia.org/other-news/30413

\section{Meetings 2023}

\section{Microscopy \& Microanalysis 2023}

July 24-28, 2023

Minneapolis, MN

www.microscopy.org

\section{Meetings 2024}

Microscopy \& Microanalysis 2024

July 28-August 1, 2024

Cleveland, $\mathrm{OH}$

www.microscopy.org

\section{CAMBRIDGE}

\section{New to the Advances in Microscopy and Microanalysis book series!}

\section{Scanning Electron} Microscopy for the

\section{Life Sciences}

Heide Schatten

University of Missouri, Columbia

US\$120.00: Hb: 978-0-521-19599-7: $312 \mathrm{pp}$

Recent developments in scanning electron microscopy (SEM) have resulted in a wealth of new applications for cell and molecular biology, as well as related biological

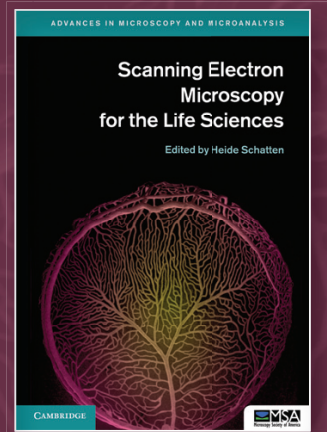

disciplines. It is now possible to analyze macromolecular complexes within their three-dimensional cellular microenvironment in near native states at high resolution, and to identify specific molecu les and their structural and molecular interactions. New approaches include cryo-SEM applications and environmental SEM (ESEM), staining techniques and processing applications combining embedding and resin-extraction for imaging with high resolution SEM, and advances in immuno-labeling. With chapters written by experts, this guide gives an overview of SEM and sample processing for SEM, and highlights several advances in cell and molecular biology that greatly benefited from using conventional, cryo, immuno, and high-resolution SEM.

\section{About the series}

The Press currently publishes the Microscopy and Microanalysis (MAM) journal in conjunction with the MSA, which reaches 4,000 microscopists and is affiliated with 12 international microscopy societies. The series would be a natural development from this journal, and will take a broad view of the discipline, covering topics from instrumentation to imaging, methodology and analysis across physical science, materials science, biology and medicine. Books commissioned for the series will range from advanced undergraduate textbooks through to research and practitioner oriented monographs for researchers. The series aims to produce a coherent source of material, encouraging the communication and exchange of ideas across these divergent fields, ensuring that the series appeals to a broad community in the physical and life sciences.

\section{Forthcoming titles in this series:}

Microscopic Nanocharacterization of Materials

by Michael Isaacson

Energy Filtered Electron Microscopy and Electron Spectroscopy by Richard Leapman

Dynamic Transmission Electron Microscopy

by Nigel Browning, Thomas LaGrange, Bryan Reed, Henning Stahlberg, Bradley Siwick 


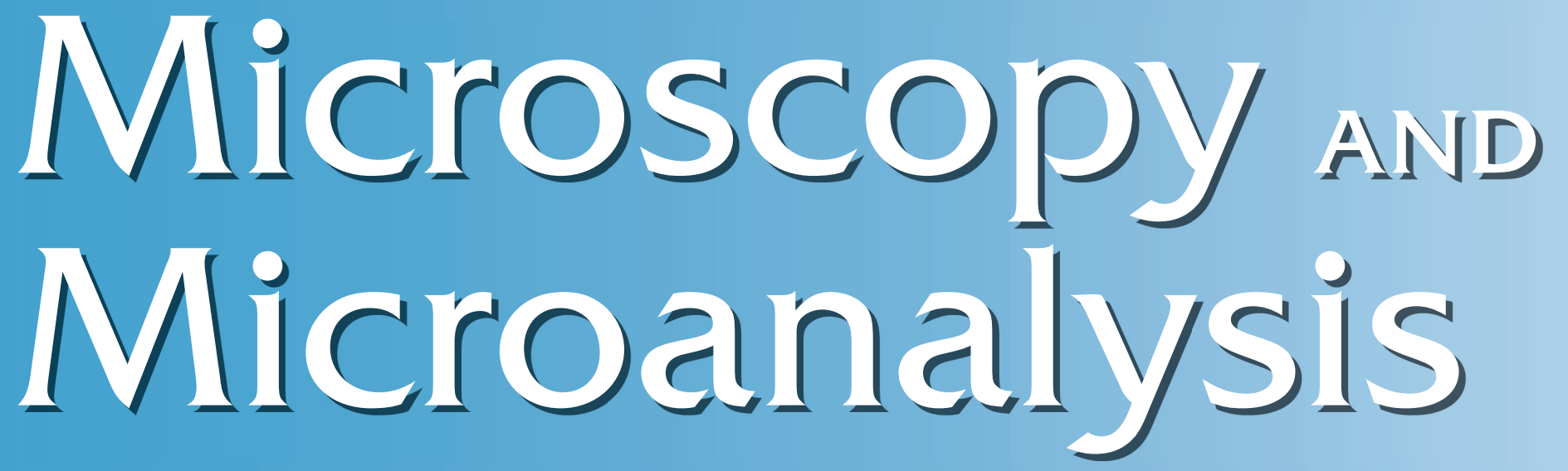

2.673

2018 Impact Factor

1 out of 9 Microscopy Journals

2018 Journal Citation Reports

(C) Clarivate Analytics

Published for the

Microscopy Society of America

Editor Dr John Mansfield

Microscopy and Microanalysis publishes

original research papers in the fields of

microscopy, imaging, and compositional

analysis. This distinguished international

forum is intended for microscopists in both

biology and materials science.

Online submission at

http://mc.manuscriptcentral.com/mam

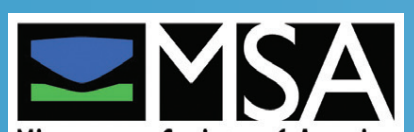

Microscopy Society of America

\section{cambridge.org/mam}
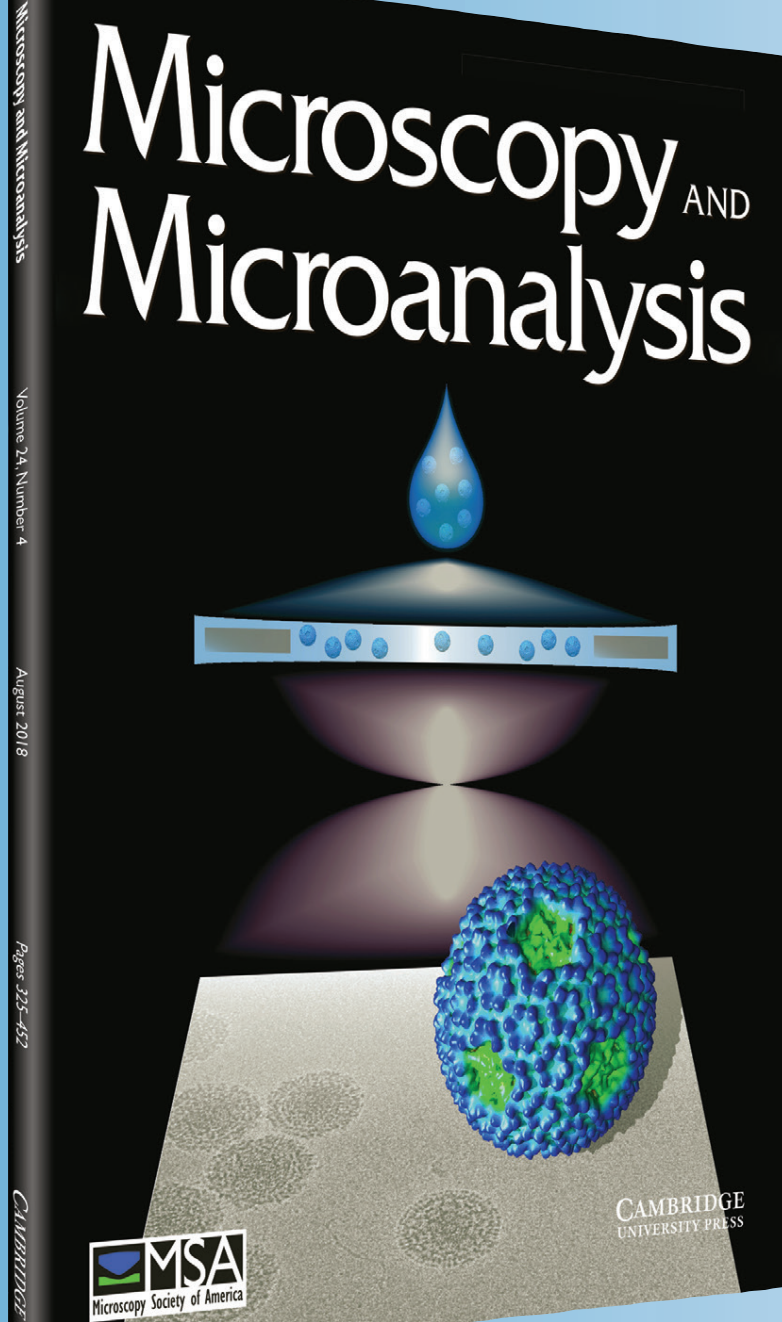

\section{पist}

